\title{
Estudio comparativo de laringes humanas y de cerdos: Hacia un modelo de entrenamiento quirúrgico
}

\section{Comparative study of human and pig larynxes: Towards a surgical training model}

\author{
Cristián Papuzinski $A^{1}$, Roberto Garnham $\mathrm{P}^{2}$, Felipe Gauna $\mathrm{P}^{2}$, Daniel Sotomayor $\mathrm{J}^{2}$, Carlos Lozano $\mathrm{B}^{3}$, \\ Francisco Tocornal $\mathrm{J}^{4}$.
}

\section{RESUMEN}

Introducción: La laringe de cerdo doméstico tiene similitudes con la laringe humana. La capacidad elástica de las cuerdas vocales del cerdo demuestran tener la mayor similitud con la humana comparado con otros animales por lo que se ha propuesto usar las laringes de cerdo como modelo de entrenamiento quirúrgico.

Objetivo: Determinar las diferencias y similitudes anatómicas e histológicas entre la laringe del cerdo y la humana.

Material y método: Se realizaron mediciones por 4 observadores en 5 laringes porcinas cuyos resultados se compararon con los descritos en la literatura para las laringes humanas. Además se realizaron cortes histológicos para visualizar fibras elásticas, mucinas neutras y mucinas ácidas.

Resultados: El cartílago tiroides porcino mide entre las astas superiores $37,55 \pm 7,30$ $\mathrm{mm}$, entre astas inferiores $31,33 \pm 3,27 \mathrm{~mm}$, desde la prominencia laríngea al borde posterior 34,32 $\pm 7,30 \mathrm{~mm}$. En el cartílago cricoides, desde el borde superior-inferior en el arco anterior 7,28 $\pm 2,21 \mathrm{~mm}$, altura borde superior-inferior pared posterior 27,47 $\pm 3,40$ $\mathrm{mm}$, ancho máximo pared posterior $30,99 \pm 4,51 \mathrm{~mm}$, diámetro interior anteroposterior (borde cefálico) 30,90 $\pm 2,12 \mathrm{~mm}$, diámetro interior anteroposterior (borde caudal) 21,78 $\pm 2,55 \mathrm{~mm}$, diámetro interior derecha-izquierda (borde cefálico) $18,11 \pm 2,13 \mathrm{~mm}$, diámetro interior derecha-izquierda (borde caudal) 21,10 $\pm 2,40 \mathrm{~mm}$. Histológicamente, la laringe de cerdo y humana presentan leves diferencias en cuanto al epitelio de cada porción de la laringe, a pesar de que el tipo de cartílago es el mismo en ambas especies.

Conclusión: Si bien existen diferencias anatómicas e histológicas entre la laringe de cerdo y el humano, el modelo porcino es una alternativa útil, accesible y de bajo costo para el entrenamiento en cirugía laringotraqueal y microcirugía laríngea.

Palabras clave: Laringe porcina, cartílago tiroides, entrenamiento quirúrgico, microcirugía laríngea.

\footnotetext{
1 Centro Interdisciplinario de Estudios en Salud (CIESAL), Universidad de Valparaíso, Chile.

2 Universidad de Valparaíso, Chile.

3 Hospital Carlos Van Buren, Valparaíso, Chile.

4 Clínica Las Condes, Santiago, Chile.

Los autores declaran no tener conflictos de interés.
}

Recibido el 20 de junio de 2019. Aceptado el 15 de agosto de 2019. 


\section{ABSTRACT}

Introduction: The domestic pig larynx has similarities with the human larynx. The elastic capacity of the vocal folds of the pig has the greater similarity with the human one compared with other animals. It has been proposed to use the porcine larynx as a model for surgical training.

Aim: To determine the anatomical and histological differences and similarities between the pig larynx and the human larynx.

Material and method: Measurements were made by 4 observers in 5 porcine larynxes whose results were compared with those described in the literature. In addition, histological sections were performed to visualize elastic fibers, neutral mucins and acid mucins.

Results: The porcine thyroid cartilage measured $37.55 \pm 7.30 \mathrm{~mm}$ between the upper horns, $31.33 \pm 3.27 \mathrm{~mm}$ between lower horns and $34.32 \pm 7.30 \mathrm{~mm}$ from the laryngeal prominence to the posterior margin. In the cricoid cartilage, from the upper-lower edge in the anterior arch $7.28 \pm 2.21 \mathrm{~mm}$, height upper-lower edge posterior wall $27.47 \pm 3.40$ $\mathrm{mm}$, maximum posterior wall width $30.99 \pm 4.51 \mathrm{~mm}$, anteroposterior inner diameter (head margin) $30.90 \pm 2.12 \mathrm{~mm}$, inner diameter anteroposterior (caudal edge) 21.78 $\pm 2.55 \mathrm{~mm}$, inner diameter right-left (head edge) $18.11 \pm 2.13 \mathrm{~mm}$, inner diameter rightleft (caudal edge) $21.10 \pm 2.40 \mathrm{~mm}$. Histologically, the pig and human larynxes present slight differences in the epithelium of each portion of the larynx, despite the fact that type of cartilage is the same in both species.

Conclusions: Although there are anatomical and histological differences between the pig larynx and the human larynx, the porcine model is a useful, accessible and low cost alternative for training in laryngotracheal surgery and laryngeal microsurgery.

Key words: Porcine larynx, thyroid cartilage, surgical training, laryngeal microsurgery.

\section{INTRODUCCIÓN}

Laringes de diferentes animales han sido utilizadas con fines académicos para evaluar aspectos tanto anatómicos como fisiológicos ${ }^{1,2}$. Por más de dos siglos la laringe del perro ha sido usada como base en estudios de fonación ${ }^{1}$. En el año 2000 Garret y cols, intentaron determinar el modelo animal más adecuado para estudios experimentales sobre cirugía de cuerdas vocales y su función mediante una comparación histológica y videoestroboscópica de la laringe en perros, monos y cerdos ${ }^{1,3}$. Llegaron a la conclusión que, basándose en los resultados histológicos y estroboscópicos, el perro era el modelo animal más adecuado para los estudios sobre cirugía de las cuerdas vocales y de fonación, reconociendo además, que la anatomía laríngea de ningún animal es idéntica a la laringe humana ${ }^{1-4}$.

Sin perjuicio de lo anterior, hay pruebas que la laringe de cerdo doméstico (Sus scrofa domestica) tiene ventajas sobre la laringe canina como modelo de fonación ${ }^{4}$. Estudios de capacidad elástica de las cuerdas vocales del cerdo demuestran tener la mayor similitud con la humana comparado con otros animales, además de tener frecuencias de ondas fonatorias similares con los humanos ${ }^{5}$. La gran dificultad con el estudio de fonación en laringes de cerdo es la realización de la laringoscopía, que es muy compleja en comparación con otros animales, ya que las cuerdas vocales falsas son muy prominentes, lo que imposibilita ver la onda de la mucosa in vivo para realizar estudios en este ámbito ${ }^{6}$.

Por otro lado, la comunidad médica ha aceptado a los cerdos como un excelente modelo para las pruebas quirúrgicas en función de la similitud de sus respectivos órganos con los humanos ${ }^{7}$. La posibilidad de obtener cerdos genéticamente modificados amplió aún más su potencial biomédico, especialmente para imitar enfermedades humanas y establecer tratamientos experimentales ${ }^{7}$. Al existir un animal que es similar anatómicamente a los 
humanos como lo es el cerdo, se ha planteado la posibilidad de simular habilidades quirúrgicas, en base a modelos animales ${ }^{8}$. La simulación es un método de enseñanza interactivo, que recrea en su totalidad o en parte una experiencia clínica, sin exponer a los pacientes a riesgos asociados ${ }^{8}$. La simulación intenta reducir resultados no deseados, proporcionando educación basada en el aprendizaje activo en un entorno de bajo riesgo, mejorando el conocimiento, la técnica y las habilidades no técnicas ${ }^{8}$. Las modalidades de simulación pueden variar según el tipo de tecnología utilizadå ${ }^{\text {. }}$ Entre los simuladores de baja tecnología, están los modelos basados en animales y en cadáveres humanos 0 animales ${ }^{8}$.

\section{OBJETIVO}

Teniendo presente lo anterior, para proponer la laringe porcina como una herramienta de simulación quirúrgica, la presente investigación tiene como objetivo determinar las diferencias y similitudes anatómicas e histológicas de la laringe del cerdo con la humana.

\section{MATERIAL Y MÉTODO}

El estudio se realizó en laringes porcinas de especímenes adultos de peso promedio. Las laringes utilizadas fueron obtenidas de un matadero, donde se faenan porcinos rutinariamente, por lo que la aprobación de bioseguridad animal no fue necesaria. Cinco laringes fueron fijadas en formalina y se realizó disección de las piezas, para observación de la irrigación, inervación y musculatura. Luego se continuó la disección, dejando expuesto exclusivamente los cartílagos tiroides, cricoides y aritenoides. Se realizó medición manual, con una regla milimétrica metálica, por 4 operadores entre diferentes puntos anatómicos de los principales cartílagos. Para los cartílagos tiroides y cricoides se consideraron 7 medidas, mientras que para el aritenoides 3 medidas. Cartílago tiroides: entre astas superiores, entre astas inferiores, cuerno superior-inferior (izquierdo), cuerno superiorinferior (derecho), escotadura tiroidea anteriorborde inferior, prominencia laríngea-borde posterior, anteroposterior en la base del cartílago. Cartílago cricoides: altura borde superior-inferior arco anterior, altura borde superior-inferior pared posterior, ancho máximo pared posterior, diámetro interior anteroposterior (borde cefálico), diámetro interior anteroposterior (borde caudal), diámetro interior derecha-izquierda (borde cefálico), diámetro interior derecha-izquierda (borde caudal). Cartílago aritenoides: proceso vocal-proceso muscular, proceso vocal-ápex, proceso muscularápex. Se realizaron cortes histológicos y tinción de dichos cortes para visualizar fibras elásticas con tinción de Verhoeff-Van Gieson, mucinas neutras con PAS y mucinas ácidas con azul de toluidina y azul de Alción.

\section{RESULTADOS}

En la anatomía macroscópica, las mediciones obtenidas fueron las siguientes:

Cartílago tiroides: Entre astas superiores 37,5 $\pm 7,30 \mathrm{~mm}$, entre astas inferiores $31,33 \pm 3,27 \mathrm{~mm}$, cuerno superior-inferior (izquierdo) 47,17 $\pm 3,87$ $\mathrm{mm}$, cuerno superior-inferior (derecho) 45,22 $\pm 7,90 \mathrm{~mm}$, escotadura tiroidea anterior-borde inferior 19,77 $\pm 1,59 \mathrm{~mm}$, prominencia laríngeaborde posterior $34,32 \pm 7,30 \mathrm{~mm}$, anteroposterior en la base del cartílago 27,30 $\pm 1,59 \mathrm{~mm}$.

Cartílago cricoides: Altura borde superior-inferior arco anterior 7,28 $\pm 2,21 \mathrm{~mm}$, altura borde superior-inferior pared posterior $27,47 \pm 3,40 \mathrm{~mm}$, ancho máximo pared posterior $30,99 \pm 4,51 \mathrm{~mm}$, diámetro interior anteroposterior (borde cefálico) $30,90 \pm 2,12 \mathrm{~mm}$, diámetro interior anteroposterior (borde caudal) 21,78 $\pm 2,55 \mathrm{~mm}$, diámetro interior derecha-izquierda (borde cefálico) 18,11 $\pm 2,13$ $\mathrm{mm}$, diámetro interior derecha-izquierda (borde caudal) $21,10 \pm 2,40 \mathrm{~mm}$.

Cartílago aritenoides: Proceso vocal-proceso muscular 12,90 $\pm 0,91 \mathrm{~mm}$, proceso vocal-ápex 16,31 $\pm 1,18 \mathrm{~mm}$, proceso muscular-ápex $17,45 \pm 1,25$ $\mathrm{mm}$. Los principales hallazgos macroscópicos se evidencian en la Figura 1 y las características histológicas se resumen en la Figura 2. En la Figura 3 se muestran los cartílagos tiroideos con sus 


\begin{tabular}{|c|c|c|c|c|}
\hline & Epiglotis & Supraglotis & Ventrículo & Subglotis \\
\hline & 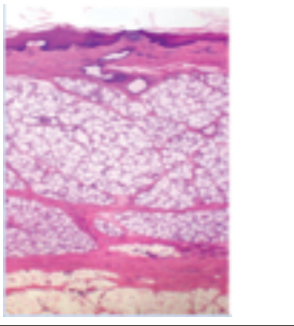 & 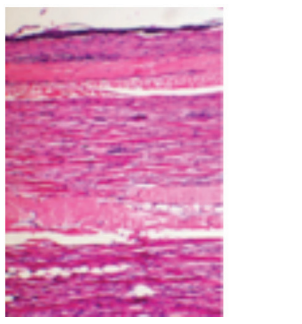 & 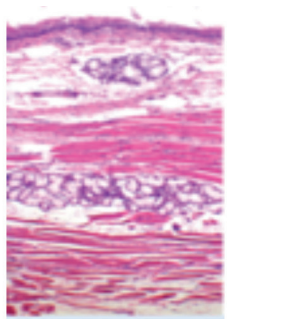 & 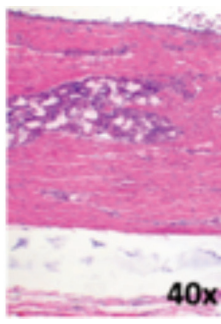 \\
\hline Epitelio & $\begin{array}{l}\text { Escamoso } \\
\text { queratinizante }\end{array}$ & $\begin{array}{l}\text { Escamoso no } \\
\text { queratinizante }\end{array}$ & $\begin{array}{l}\text { Respiratorio alto } \\
\text { de } 8 \text { capas }\end{array}$ & $\begin{array}{l}\text { Respiratorio bajo } \\
\text { de } 4 \text { capas }\end{array}$ \\
\hline $\begin{array}{l}\text { Tejidos parietales } \\
\text { (profundidad) }\end{array}$ & $\begin{array}{l}\text { Epitelio, tejido conectivo } \\
\text { denso, glándulas mucinosas, } \\
\text { tejido adiposo }\end{array}$ & $\begin{array}{l}\text { Epitelio, tejido conectivo } \\
\text { denso, musculatura } \\
\text { estriada en estroma conectivo } \\
\text { denso y abundante } \\
\text { vascularización venosa }\end{array}$ & $\begin{array}{l}\text { Epitelio, tejido conectivo laxo } \\
\text { con glándulas seromucinosas, } \\
\text { musculatura estriada }\end{array}$ & $\begin{array}{l}\text { Epitelio, tejido conectivo } \\
\text { denso con glándulas } \\
\text { seromucinosas }\end{array}$ \\
\hline $\begin{array}{l}\text { Tejido } \\
\text { predominante }\end{array}$ & Glándulas mucinosas & $\begin{array}{l}\text { Musculatura estriada en } \\
\text { estroma conectivo denso }\end{array}$ & Musculatura estriada & $\begin{array}{l}\text { Tejido conectivo } \\
\text { denso }\end{array}$ \\
\hline
\end{tabular}

Figura 1. Principales hallazgos histológicos en cortes de laringe de cerdo.

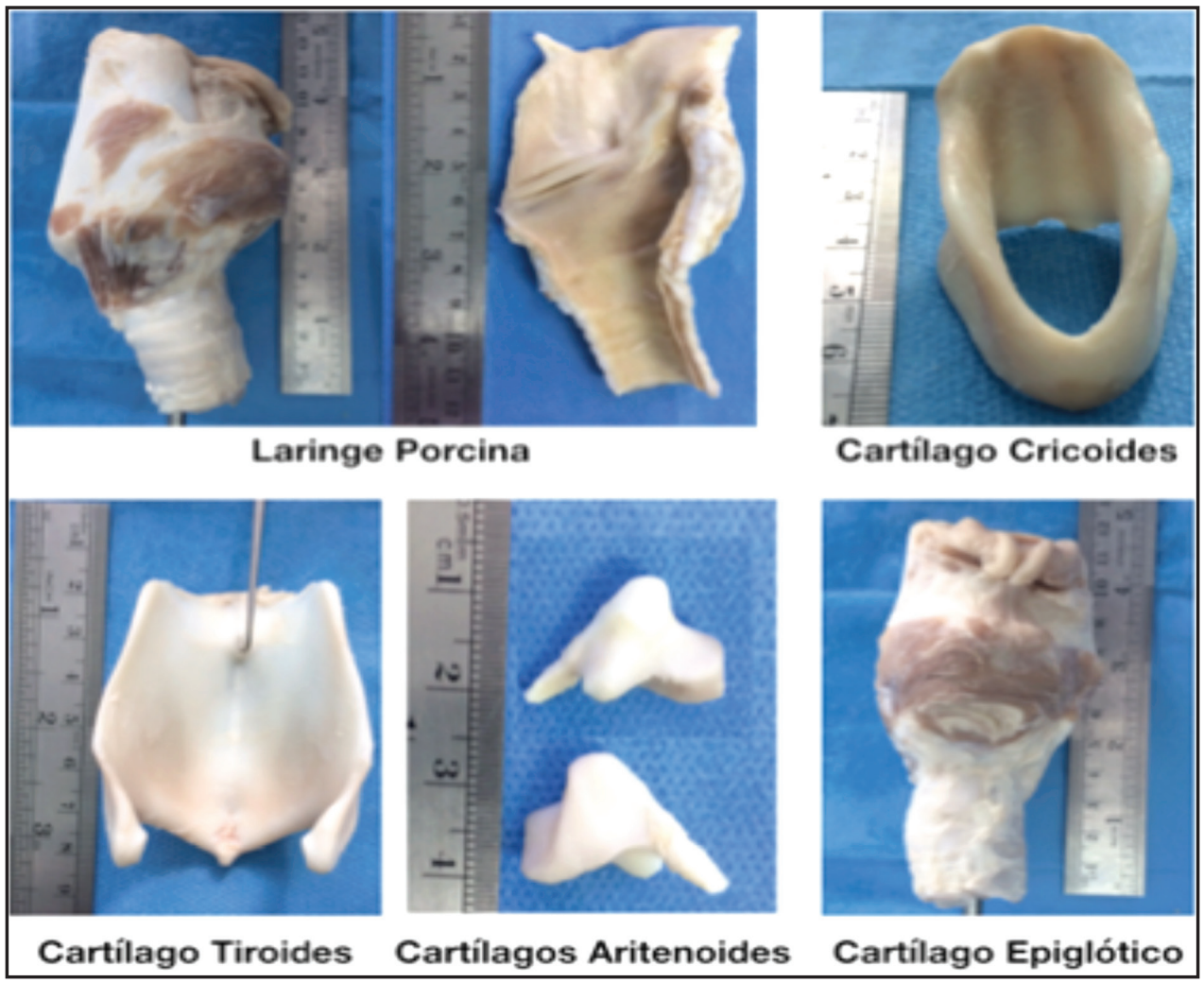

Figura 2. Estructura macroscópica de los cartílagos laríngeos porcinos. 


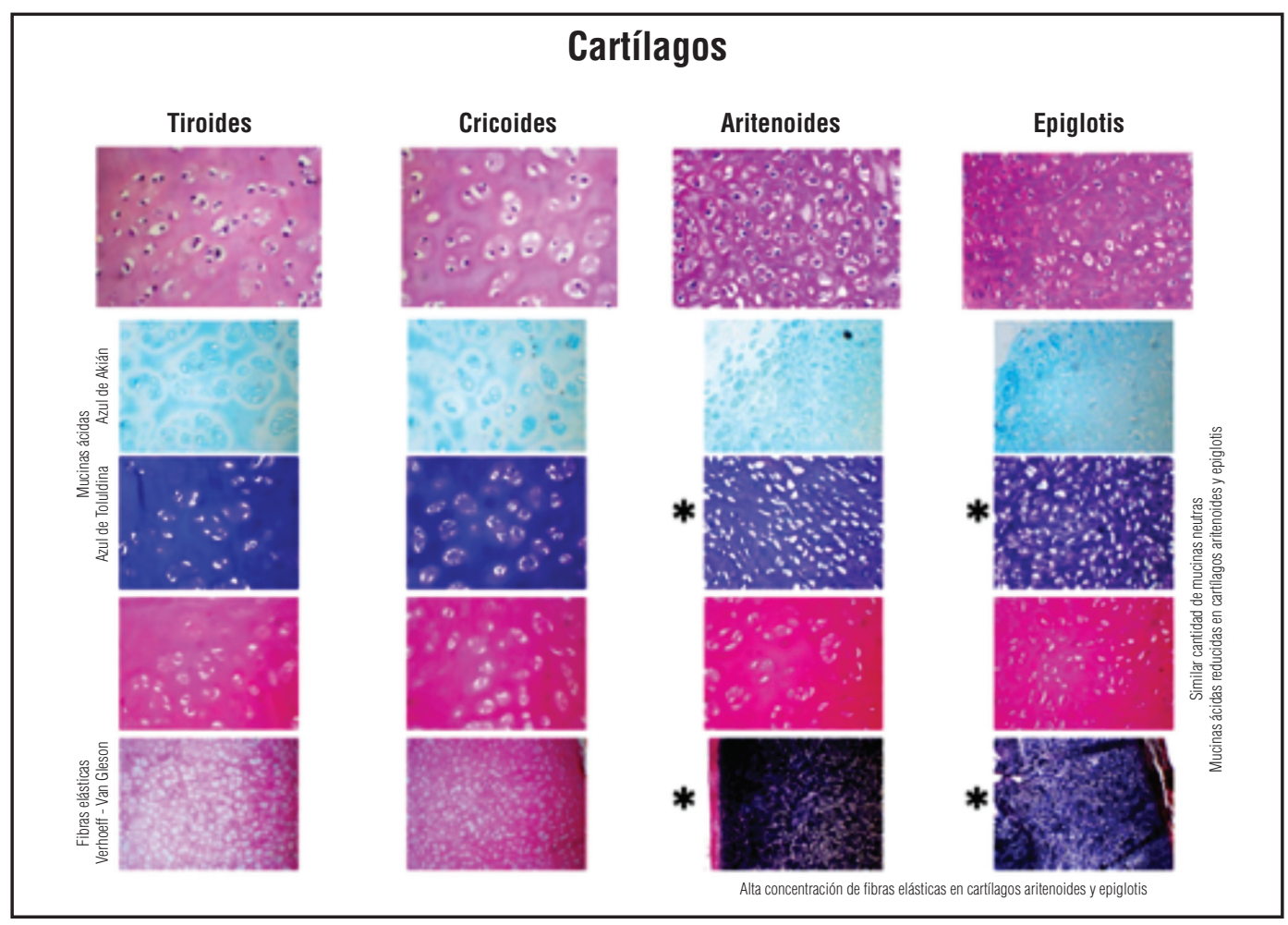

Figura 3. Cortes histológicos de cartílagos laríngeos porcinos.

respectivos cortes histológicos y sus tinciones correspondientes. Destaca la similar cantidad de mucinas neutras y mucinas ácidas reducidas en los cartílagos aritenoides y epiglotis.

\section{DISCUSIÓN}

\section{Estructura laríngea}

La laringe porcina es una estructura tubular similar a la laringe humana. Sus paredes están constituidas de cartílago, pericondrio, músculo estriado, tejido conjuntivo y mucosa ${ }^{2,9}$. Los espacios de la endolaringe se pueden dividir, al igual que en el hombre, en área supraglótica, incluyendo a la epíglotis, los ventrículos, el vestíbulo y las bandas ventriculares; área glótica, incluidas los pliegues vocales; área infraglótica, ubicada inferior a la glotis, hasta llegar a la base del cartílago cricoides $^{1,9}$. El marco cartilaginoso también es muy similar al de los humanos, pero de tamaño ligeramente mayor ${ }^{9}$. Los cartílagos únicos son el cricoides, la epíglotis y el cartílago tiroides. Los cartílagos pares son los cartílagos aritenoides y corniculados ${ }^{9}$.

\section{Cartílago tiroides}

El cartílago tiroides recibe su nombre por su forma de escudo (del griego thureoeides: en forma de escudo ${ }^{10}$. En humanos, se sitúa bajo el hueso hioides y constituye un escudo protector de la laringe. Está formado por dos láminas laterales cuadriláteras 0 alas, que están unidas entre sí al nivel anterior en la línea media, formando un ángulo agudo llamado prominencia laríngea ${ }^{10}$. La unión es incompleta a nivel superior, donde las láminas están separadas por la escotadura tiroidea superior ${ }^{10}$. Cabe destacar, que en las láminas laríngeas existe un borde posterior vertical prolongado en sentido superior llamado asta superior de aproximadamente 15 a $20 \mathrm{~mm}$ de largo, que articula con el hueso hioides mediante la membrana tirohioidea y en 
sentido inferior, la lámina se prolonga con el asta inferior, que presenta una superficie articular con el cricoides ${ }^{10}$.

El cartílago tiroides en cerdos tiene forma de canoa y estructura la mayor parte del esqueleto laríngeo del animal11. La gran diferencia con el humano es que la asta superior está ausente y no posee escotadura tiroidea ${ }^{12}$. El ángulo caudodorsal es prolongado, formando un asta inferior ancha y corta que articula con el cricoides ${ }^{11}$. Por otro lado, la línea oblicua en la cara lateral del cartílago, lugar de inserción del músculo tirohioideo, constrictor de la faringe y esternohioideo, está ausente en la mayoría de los $\operatorname{cas}^{12}{ }^{12}$. Las láminas flanquean los cartílagos aritenoides y forman gran parte de la pared lateral laríngea ${ }^{11}$ (Tabla 1).

\section{Cartílago cricoides}

El cartílago cricoides (del griego krikoeides: en forma de anillo), en humanos se sitúa en la parte inferior de la laringe y tiene forma de anillo de sello, con el sello a nivel posterior y un anillo 0 arco anterior ${ }^{10}$. Tiene un arco anterolateral con un tubérculo cricoideo medial y, a nivel posterior, una placa o sello cricoideo, con dos superficies articulares aritenoideas superolaterales y dos superficies articulares tiroideas inferolaterales ${ }^{10}$. El borde inferior del cartílago es irregular y suele estar unido lateralmente al primer anillo traqueal ${ }^{10}$. El borde superior está unido a nivel anterior a la membrana cricotiroidea y a ambos lados al músculo cricoaritenoideo lateral ${ }^{10}$. La cara posterior del sello está dividida por una cresta roma, medial y vertical (la lámina posterior), en dos superficies laterales deprimidas, en las que se insertan los músculos cricoaritenoideos posteriores ${ }^{10}$. El cartílago cricoides es elíptico en los cerdos, en lugar de tener la forma de "anillo de sello" característica de los humanos. Se ubica rostral al primer anillo traqueal ${ }^{11}$. La placa cricoidea es alta y tiene una cresta distinta a la de los humanos además de ser afilada en su superficie posterior ${ }^{12,14}$ (Tabla 2).

\section{Cartílago aritenoides}

El cartílago aritenoides (del griego arytainoeides: en forma de aceitera o cántaro) tiene forma de pirámide triangular que presenta tres caras, una base que se articula con el cricoides y un vértice

Tabla 1. Mediciones del cartílago tiroides humano y porcino $(n=7)$. Bakhshaee y cols ${ }^{12}$

\begin{tabular}{|lccc|}
\hline Distancias promedio $(\mathrm{mm}) \pm \mathrm{DS}$ & Humanos & Cerdos & Diferencia de medias \\
\hline Entre astas superiores & $37,55 \pm 7,30$ & $33,8 \pm 2,14$ & 3,75 \\
Entre astas inferiores & $31,33 \pm 3,27$ & $31,7 \pm 2,17$ & $-0,37$ \\
Cuerno superior-inferior (izquierdo) & $47,17 \pm 3,87$ & $58 \pm 3,65$ & $-10,83$ \\
Cuerno superior-inferior (derecho) & $45,22 \pm 7,90$ & $57,85 \pm 3,45$ & $-12,63$ \\
Escotadura tiroidea anterior-borde inferior & $19,77 \pm 1,59$ & $61,7 \pm 1,71$ & $-41,93$ \\
Prominencia laríngea-borde posterior & $34,32 \pm 7,30$ & $33,65 \pm 1,98$ & 0,67 \\
Anteroposterior en la base del cartílago & $27,30 \pm 1,59$ & $41,6 \pm 2,13$ & $-14,3$ \\
\hline
\end{tabular}

Tabla 2. Mediciones del cartílago cricoides humano y porcino $(n=8)$. Bakhshaee y $\operatorname{cols}^{12}$

\begin{tabular}{|lccc|}
\hline Distancia promedio $(\mathrm{mm}) \pm$ DS & Humanos & Cerdos & Diferencia de medias \\
\hline Altura borde superior-inferior arco anterior & $7,28 \pm 2,21$ & $8,8 \pm 0,95$ & $-1,52$ \\
Altura borde superior-inferior pared posterior & $27,47 \pm 3,40$ & $26,75 \pm 3,24$ & 0,72 \\
Ancho máximo pared posterior & $30,99 \pm 4,51$ & $26,8 \pm 3,1$ & 4,19 \\
Diámetro interior anteroposterior (borde craneal) & $30,90 \pm 2,12$ & $44,95 \pm 1,43$ & $-14,05$ \\
Diámetro interior anteroposterior (borde caudal) & $21,78 \pm 2,55$ & $32,3 \pm 1,3$ & $-10,52$ \\
Diámetro interior derecha-izquierda (borde craneal) & $18,11 \pm 2,13$ & $19,75 \pm 2,61$ & $-1,64$ \\
Diámetro interior derecha-izquierda (borde caudal) & $21,10 \pm 2,40$ & $19,4 \pm 1,42$ & 1,7 \\
\hline
\end{tabular}


libre ${ }^{10}$. La base del cartílago aritenoides es cóncava y se articula con la superficie superolateral de la lámina del cartílago cricoides, el ángulo lateral se llama proceso musculary el ángulo anterior proceso vocal ${ }^{15}$. El vértice se articula con un cartílago corniculado ${ }^{15}$. La superficie medial de cada cartílago se enfrenta con la otra ${ }^{15}$. La superficie anterolateral tiene dos depresiones para la inserción de los músculos vocales y el ligamento vestibular ${ }^{15}$. En el ángulo anterior se ubica la apófisis vocal donde se une con los ligamentos vocales ${ }^{15}$. El cerdo presenta un cartílago interaritenoideo entre los cartílagos aritenoides, que cierra completamente el área aritenoidea $^{12}$. El cartílago aritenoideo del cerdo tiene un largo proceso vocal y un proceso muscular más corto ${ }^{12}$ (Tabla 3 ).

\section{Cartílago epiglótico}

El cartílago epiglótico (del griego epiglottis: pequeña lengua) tiene forma de hoja y se sitúa en la parte anterosuperior de la laringe, por detrás del cartílago tiroides, al que sobrepasa en sentido superior ${ }^{10}$. Se inserta por anterior, en el ángulo entrante del cartílago tiroides mediante el ligamento tiroepiglótico ${ }^{10}$. La epiglotis presenta una cara laríngea posteroinferior que tiene muchas depresiones cribiformes, una cara lingual anterosuperior cóncava orientada en sentido anterosuperior, así como una base superior y dos bordes laterales ${ }^{10}$. Cubre el margen laríngeo durante la deglución por un movimiento pasivo ${ }^{10}$. El cartílago epiglótico en cerdos, es de considerable tamaño; tiene una forma tubular con el extremo frontal y es curvado en el exterior ${ }^{12}$. También se caracteriza por ubicarse en la parte posterosuperior de la laringe y por la falta de un vértice evidente, su pedículo es corto y redondeado ${ }^{12}$.

\section{Inervación laríngea}

En humanos el nervio laríngeo recurrente (NLR) emerge desde el nervio vago ${ }^{14}$. Cerca del arco aórtico, en el área del mediastino, el nervio vago izquierdo se ramifica para formar el nervio laríngeo recurrente izquierdo (NLRI) que rodea el arco aórtico inferior y alcanza el surco entre la tráquea y el esófago, para luego entrar en la laringe entre el cartílago cricoides y el cartílago tiroides penetrando al músculo cricoaritenoideo lateral ${ }^{14}$. En el lado derecho, cerca de la arteria subclavia, el nervio vago da lugar al nervio laríngeo recurrente derecho (NLRD), en el cual, de la misma manera sucede en el NLRI se extiende a lo largo del surco traqueoesofágico, llega a la laringe y de ahí se dirige al músculo cricoaritenoideo posterior ${ }^{14}$. Es importante destacar que los nervios laríngeos recurrentes inervan todos los músculos intrínsecos de la laringe, a excepción del músculo cricotiroideo, éste recibe una rama externa del nervio laríngeo superior ${ }^{14}$.

Los nervios laríngeos del cerdo tienen la misma fuente, ramificación y distribución que presenta la inervación laríngea humana ${ }^{14}$. Al igual que los humanos, el NLRI, inmediatamente después de ramificarse desde el nervio vago, pasa por debajo del arco aórtico y alcanza el área de unión traqueoesofágica, donde se eleva ${ }^{14}$. El NLRD se bifurca y pasa debajo de la arteria subclavia derecha, también se eleva desde la unión traqueoesofágica ${ }^{14}$.

\section{Irrigación laríngea}

El suministro sanguíneo de la laringe humana consiste en dos pares de venas y arterias a cada lado ${ }^{15}$. La irrigación arterial está dada por las

Tabla 3. Mediciones del cartílago aritenoides humano y porcino $(n=6)$. Bakhshaee y cols ${ }^{12}$

\begin{tabular}{|lccc|}
\hline $\begin{array}{l}\text { Distancia } \\
\text { Promedio }(\mathrm{mm}) \pm \text { DS }\end{array}$ & Humanos & Cerdos & Diferencia de medias \\
\hline Proceso vocal - proceso muscular & $12,90 \pm 0,91$ & $14,95 \pm 0,94$ & $-2,05$ \\
Proceso vocal - ápex & $16,31 \pm 1,18$ & $15,45 \pm 1,39$ & 0,86 \\
Proceso muscular - ápex & $17,45 \pm 1,25$ & $14,6 \pm 1,09$ & 2,85 \\
\hline
\end{tabular}


arterias laríngeas superior e inferior que derivan de las arterias tiroideas superior e inferior, estas últimas son ramas de las arterias carótida y subclavia respectivamente ${ }^{15}$. Las venas laríngeas superior e inferior van paralelas a las arterias ${ }^{15}$. Las principales arterias que suministran la laringe porcina son la arteria laríngea craneal que surge de la arteria carótida común, cerca de su terminación, y la arteria tiroidea caudal que surge de la arteria braquiocefálica bilateralmente ${ }^{9}$. El drenaje venoso es hacia la vena yugular interna a través de la vena laríngea craneal y hacia la vena yugular externa a través de las venas laríngeas caudales ${ }^{9}$. Además, hay un drenaje venoso sustancial a través de las venas tiroideas caudales directamente en las venas braquiocefálica ${ }^{9}$.

\section{Musculatura laríngea}

Los músculos con fijación en la laringe se pueden clasificar como extrínsecos 0 intrínsecos ${ }^{14}$. Los músculos extrínsecos tienen un punto de unión en el exterior y otra inserción en los cartílagos laríngeos $^{14}$. Este grupo de músculos es parte del sistema suspensorio de la laringe ${ }^{14}$. Los músculos intrínsecos tienen todas sus inserciones ubicadas en las estructuras de la laringe ${ }^{14}$. Estos músculos son responsables de los movimientos articulatorios entre los cartílagos. Incluyen, en la pared externa de la laringe: el músculo cricotiroideo, y en los espacios internos: los músculos interaritenoideos, tiroaritenoideo, cricoaritenoideo vocal, posterior y lateral ${ }^{14}$. En el cuerpo de cada cuerda vocal, es posible encontrar el músculo vocal ${ }^{14}$. El músculo vocal fue considerado, antes de la Nómina Anatómica de 1998, como la porción interna del músculo tiroaritenoideo, pero hoy en día, se clasifica como un músculo independiente ${ }^{14}$. Es importante enfatizar que en los cerdos, los músculos intrínsecos son similares a los músculos laríngeos humanos ${ }^{9}$. La única diferencia está en el músculo interaritenoideo, que es más voluminoso en los cerdos que en humanos ${ }^{9}$.

\section{Anatomía microscópica}

En humanos, la epiglotis y las cuerdas vocales verdaderas presentan un epitelio escamoso estratificado no queratinizado. Las cuerdas vocales falsas, el ventrículo y la subglotis posee epitelio respiratorio pseudoestratificado ciliado ${ }^{16}$. Existe un epitelio transicional entre el epitelio respiratorio pseudoestratificado ciliado de la supraglotis y el epitelio escamoso estratificado de las cuerdas vocales verdaderas ${ }^{16}$. Este epitelio transicional aparece abruptamente y está compuesto de células escamosas inmaduras con hipercelularidad, y aumento de la razón núcleo citoplasma ${ }^{16}$. Las glándulas seromucosas se encuentran en los dos tercios inferiores de la epiglotis, en la submucosa ventricular y en la subglotis ${ }^{16}$. Se encuentran ausentes en las cuerdas vocales verdaderas ${ }^{16}$.

En cerdos, la supraglotis y la glotis están compuestas por un epitelio escamoso estratificado y la subglotis por un epitelio columnar ciliado pseudoestratificado ${ }^{9}$. Se pueden observar glándulas túbulo-acinosas mixtas y células caliciformes tanto en la supraglotis como en la glotis ${ }^{9}$. Por lo tanto, la histología es similar a la laringe humana, excepto por el hecho de que la supraglotis porcina está revestida por epitelio escamoso estratificado, en contraste con la mucosa respiratoria en humanos que es epitelio respiratorio pseudoestratificado ciliado ${ }^{9}$.

\section{Estructura de las cuerdas vocales}

Hirano describe la estructura de las cuerdas vocales en humanos por primera vez en 1973, describe cinco capas estructuradas bien definidas en las cuerdas vocales ${ }^{17}$. La principal parte de las cuerdas vocales es el músculo vocal, el cual es un músculo estriado inervado por el NLR como se vio anteriormente ${ }^{17}$. Al músculo le sigue la lámina propia profunda compuesta más por fibras de colágeno, luego la lámina propia intermedia compuesta por fibras elásticas y finalmente la lámina propia superficial o espacio de Reinke ${ }^{18}$. La lámina propia es una capa continua de aproximadamente un milímetro de espesor que se envuelve alrededor del borde libre del pliegue vocal ${ }^{6}$. Espacio de Reinke es tejido conectivo laxo dentro de la lámina propia de las cuerdas vocales verdaderas, ubicada entre el ligamento de las cuerdas vocales y el epitelio escamoso de superficie ${ }^{16}$. Contiene capilares, pero no posee linfáticos. Posee escaso número de glándulas seromucosas ${ }^{16}$. Las cuerdas vocales están revestidas por un epitelio escamoso 
estratificado no queratinizado ${ }^{18}$. El ligamento de las cuerdas vocales es un tejido elástico adelgazado que yace bajo el borde libre de las cuerdas vocales ${ }^{16}$. Se inserta en el cartílago tiroides por anterior y en el proceso vocal del aritenoides por posterior ${ }^{16}$.

La estructura histológica de la cuerda vocal porcina, con su revestimiento epitelial, lámina propia y musculatura, también muestra una gran similitud con la humana ${ }^{1,2}$. Al igual que en los humanos, la lámina propia tiene aproximadamente un milímetro de espesor a lo largo del borde medial del pliegue vocal ${ }^{6}$. El colágeno se organiza de forma laxa en la lámina propia superficial y se concentra más densamente en niveles más profun$\operatorname{dos}^{6}$ (Tabla 4).

\section{Hacia un modelo experimental}

El manejo quirúrgico de las estructuras laringotraqueales requiere de un entrenamiento especial ya que las características de la vía aérea no permiten errores. Esto hace que se dificulte la enseñanza y la adquisición de competencias ${ }^{1,2}$. El entrenamiento tradicional para la cirugía laringotraqueal se lleva cabo mediante la observación y la posterior aplicación de las destrezas observadas supervisadas por profesional con mayor experiencia ${ }^{1,2}$.

Se han propuesto modelos virtuales, con base en programas informáticos y aprovechando la tecnología de los simuladores. Estos modelos son útiles pero caros y poco asequibles ${ }^{1,2}$. A su vez, los modelos inorgánicos como los maniquíes, que simulan la anatomía humana, carecen de elasticidad y su consistencia tiende a ser distinta y su uso resulta monótono ${ }^{1,2}$. En cuanto a la utilización de cadáveres humanos, aunque son los más aptos para el entrenamiento quirúrgico requieren de conservación y mantenimiento en instalaciones adecuadas, aprobación por parte de las autoridades sanitarias, de los diversos comités de las instituciones hospitalarias, así como disponibilidad de éstos ${ }^{1,2}$.

\section{CONCLUSIÓN}

$\mathrm{Si}$ bien existen diferencias anatómicas e histológicas entre la laringe de cerdo y el humano, el modelo porcino es una alternativa útil, accesible y de bajo costo para el entrenamiento en cirugía laringotraqueal y microcirugía laríngea.

Tabla 4. Principales diferencias microscópicas entre la laringe humana y la del cerdo

\begin{tabular}{|c|c|c|}
\hline Estructura & Humanos & Cerdos \\
\hline Supraglotis & Epitelio respiratorio pseudoestratificado ciliado & $\begin{array}{l}\text { Epitelio escamoso no queratinizante } \\
\text { Tejido predominante: Musculatura estriada en } \\
\text { estroma conectivo denso }\end{array}$ \\
\hline Epiglotis & $\begin{array}{l}\text { Epitelio escamoso estratificado no queratinizado. } \\
\text { Glándulas seromucosas en sus } 2 / 3 \text { inferiores }\end{array}$ & $\begin{array}{l}\text { Epitelio escamoso queratinizante } \\
\text { Glándulas mucinosas }\end{array}$ \\
\hline \multicolumn{3}{|l|}{ Glotis } \\
\hline Cuerdas vocales verdaderas & \multicolumn{2}{|l|}{$\begin{array}{l}\text { Epitelio escamoso estratificado no queratinizado } \\
\text { Sin glándulas seromucosas } \\
\text { Organización en } 5 \text { capas }\end{array}$} \\
\hline Subglotis & $\begin{array}{l}\text { Epitelio respiratorio pseudoestratificado ciliado } \\
\text { Glándulas seromucosas }\end{array}$ & $\begin{array}{l}\text { Epitelio respiratorio bajo de } 4 \text { capas, } \\
\text { Tejido conectivo denso con glándulas } \\
\text { seromucinosas }\end{array}$ \\
\hline \multicolumn{3}{|l|}{ Tipo de cartílago } \\
\hline $\begin{array}{l}\text { Tiroides } \\
\text { Cricoides } \\
\text { Aritenoides }\end{array}$ & Hialino & \\
\hline Epiglotis & Elástico & \\
\hline
\end{tabular}




\section{BIBLIOGRAFÍA}

1. Nasser M, Wahba Ha, Kamal E, El-Makhzangy AM, BahaA N. Animal model for training and improvement of the surgical skills in endolaryngeal microsurgery. J Voice 2012; 26: 351-7.

2. Propst E, Tirado Y, Abdulkader F, Estrada M, Campisi P, Forte V. Exposure and Anatomy of the Pig Airway: Comparison with the Human Airway. En: Airway Reconstruction Surgical Dissection Manual. San Diego: Plural Publishing Inc, 2014; 13-19.

3. Garrett CG, Coleman JR, Reinisch L. Comparative Histology and Vibration of the Vocal Folds: Implications for Experimental Studies in Microlaryngeal Surgery. Laryngoscope 2000; 110: 814-24.

4. Jiang JJ, Raviv JR, Hanson DG. Comparison of the Phonation-Related Structures Among Pig, Dog, White-Tailed Deer, and Human Larynges. Ann Otol Rhinol Laryngol 2001; 110: 1120-5.

5. Alipour F, Finnegan EM, Jaiswal S. Phonatory characteristics of the excised human larynx in comparison to other species. J Voice 2013; 27: 441-7.

6. Woodson G. Developing a porcine model for study of vocal fold scar. J Voice 2012; 26: 706-10.

7. Whyte JJ, Prather RS. Genetic modifications of pigs for medicine and agriculture. Mol Reprod Dev 2011; 78: 879-91.

8. Campelo F, Netto S, Zacharias P. A porcine model for teaching surgical cricothyridootomy. Rev Col Bras 2015; 42: 193-6.

9. Gorti GK, Birchall MA, Haverson K, Macchiarini P, Balley M. A Preclinical Model for Laryngeal Transplantation: Anatomy And
Mucosal Immunology of The Porcine Larynx. Transplantation 1999; 68: 1338-42.

10. Céruse P, Ltaief-Boudrigua A, Buiret $G$, Cosmidis A, Tringali S. Anatomía descriptiva, endoscópica y radiológica de la laringe. EMC Otorrinolaringología 2012; 41: 1-26.

11. HaRe W. Sistema Respiratorio de los Equinos. En Getty R, Sisson y Grossman Anatomía de Ios Animales Domésticos. Tomo II. Barcelona, $5^{\mathrm{a}}$ ed. Masson, 2000; 1414-21.

12. Wysocki J, JaniUk I, CharUta $A$. Analysis of larynx measurements and proportions in young and adult domestic pigs. Turk J Vet Anim Sci 2010; 34: 339-47.

13. Bakhinaee H, Moro C, Kost K, Mongeau L. Threedimensional reconstruction of human vocal folds and standard laryngeal cartilages using computed tomography scan data. J Voice 2013; 27: 769-77.

14. LeÃo H dLP, Rosito J, LamberTs M. Ultra Structure of the Denervated Vocal Muscle Mechanically in Hogs (Sus Scrofa Domestica). Intl Arch Otorhinolaryngol 2010; 14: 66-81.

15. Drake R, Vogl W, Mitchell A. Chapter 8: Larinx. En: Drake R, Vogl W, Mitchell A. Gray's Anatomy for Students. Philadelphia, $2^{\circ}$ ed: Elsevier; 2010; 997-1012.

16. Bruce W. Chapter 11: Anatomy of the Neck. En: Bruce W, Atlas of Head and Neck Pathology. Philadelphia, $3^{\circ}$ ed: Elsevier; 2016.

17. Hirano M. Morphological Structure of the Vocal Cord as a Vibrator and its Variations. Folia Phoniatr Logop 1974; 26: 89-94.

18. Hirano M. Structure and vibratory behavior of the vocal folds. In: Sawashima M, Franklin SC, eds. Dynamic aspects of speech production. Tokyo, Japan: University of Tokyo Press, 1977; 13-30. 